This is a forum for perspectives on designing for communities marginalized by economics, social status, infrastructure, or policies. It will discuss design methods, theoretical and conceptual contributions, and methodological engagements for underserved communities. - Nithya Sambasivan, Editor

\title{
A Framework for Technology Design for Emerging Markets
}

Nithya Sambasivan, Nibha Jain, Garen Checkley, Asif Baki, and Taylor Herr, Google

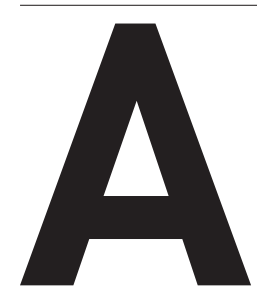

billion new people are expected to come online in the next couple of yearsalmost entirely from emerging markets. A majority of these new users will gain access through a mobile phone. Decreased costs of smartphone production, shifting localities for labor, increased rollout of Internet pipes, and aspirational desires have led to a rapid growth and uptake of the Internet in such markets.

Today, Internet software can be made available to millions across the world instantly. However, technologies often enter heterogeneous cultural, social, and economic milieus in their traversals. The HCI community can play a critical role in bringing insights on practices, values, and infrastructures in these contexts to the design process.

In this article, we share the process of the creation and dissemination of a research-led framework for designing for emerging markets, at Google and externally. In the second half, we discuss the framework, its principles, and its implementation.

\section{CREATING ORGANIZATIONAL CHANGE}

Our small group of researchers wanted to enable teams to increase their awareness of global communities and think deeply about designing technologies for them. We had conducted research for several years in emerging markets and could also draw upon a wealth of external knowledge in related fields like ICT for development (ICTD) and HCI for development (HCI4D). In most technology companies, researchers are incentivized through their localized impact on a product's trajectory, for example, via designs or features. As Google focused more energy on emerging markets, we felt the need to widely disseminate our research to all parts of the organization in order to formatively shape the vision, planning, and execution of products. The challenge ahead of us was to translate user research into the core of Google's DNA. Effective and sustainable change would come from influencing the central paradigms of software development and design.

We set forth to distill the lessons and themes from several years of field research (Figure 1). We first tied together disparate themes into a holistic framework that took into account the complexities of emerging markets, from mobile Internet connectivity to locally

Insights

$\rightarrow$ Go beyond insights by synthesizing and translating research to communities of practice.

$\rightarrow$ Frameworks can help convey technological, social, and economic aspects holistically; guidelines and tools can help act on these aspects.

$\rightarrow$ Consider the nine-dimension framework used at Google for technology design and development in emerging markets. meaningful content to social units of consumption. The framework format made the information sticky and memorable, and thus easier to rally around with various product teams. By combining different types of learning, from the technological to the sociocultural, we ensured that each community of practice had something with which they could be familiar and in which they could find comfort. Therein we had the opportunity to expose the community to other dimensions of the framework that were not directly in their wheelhouse.

Our next step was to create actionable guidelines, examples, and guiding motivations. Principles around a user community could help build empathy and intuition; however, we believed that actionability was key to organization-wide cultural change. The challenge was that we were not experts in the various domains that encompassed our framework. We therefore identified experts in each of the framework categories and built a collection of guidelines for design, code, marketing, content, and business.

For engineers. Translating the more tangible principles of the framework (Connectivity, Compatibility, and Cost) into concrete guidelines helped lower friction for the implementing engineers, enabling them to code differently. A partnership with an internal organization that led developer relations resulted in the company's first official guidance for developers and designers building for emerging markets: Building for Billions 
(https://developers.google.com/ billions/). By having the developer organization evangelize to its own community of practice, we facilitated greater trust and expertise than if we had approached them ourselves. Internally, our framework was translated into libraries to instrument and report the performance of applications for various technical dimensions, such as latency on slow connectivity, crash rates of low-end devices, and memory consumption on low-RAM resources. Corresponding metrics were developed to measure the success rates of the dimensions, such as APK size, battery usage, and crashes on simulated low-end devices.

For designers. We partnered with the design community of practice to create design specifications and metaphors for the various principles. Each technology dimension was provided with design counterparts. A few quick wins by user testing and design sprinting with products gave us confidence about the dimensions. We then disseminated the guidelines to the broader Google UX community.

For the general public. We further commoditized our framework into posters, tech talks, and digestible principles that were shared widely across the company. The dissemination exercises in turn helped us raise awareness and visibility for new communities coming online. Outside Google, several relevant tech talks have been hosted at Google I/O (Google's flagship developer conference) and Google for India, in addition to Building for Billions. The key tenets of our framework have played a major role in focusing the experiences of products such as YouTube Go, Chrome, Google Station, and others in emerging markets.

We, the HCI research community, bring great passion to advocating

\section{The framework format made the information sticky and memorable, and thus easier to rally around with various product teams.}

and helping to do the right thing for end users. Often we are comfortable operating within conventional methods, processes, and deliverables but stop short of enabling wider organizational or cultural change due to various constraints. We encourage the HCI community to synthesize digestible insights, translate these into the languages of the various communities of practice, and enable various disciplines around us to build empathy and become human-centered advocates themselves.

We now turn to our design framework. You can find a worksheet on the next page to apply the framework to your design processes.

\section{THE FRAMEWORK}

Well-designed products effortlessly add value, easily fit into people's lives, and bring a spark of joy. While the fields of ICTD and HCI have made inroads into designing technologies with care and sensitivity to sociocultural contexts, there are fewer efforts among the practitioner community to create holistic dimensions that encompass various aspects of defining, making, and implementing technologies for emerging markets. We offer a principled methodology of nine dimensions (6C's and 3S's) for product as well as research design (Figure 2).

Let's assume your research points to some promising opportunities for design in a regional context. First, can your design be accessed in the desired contexts?

Connectivity. While the Internet is on the rise in the Global South, it is still slow, unreliable, and often

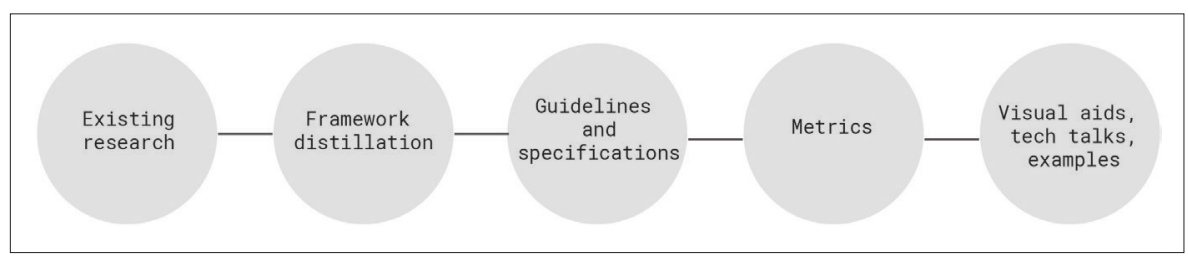

Figure 1. Framework creation and dissemination process.

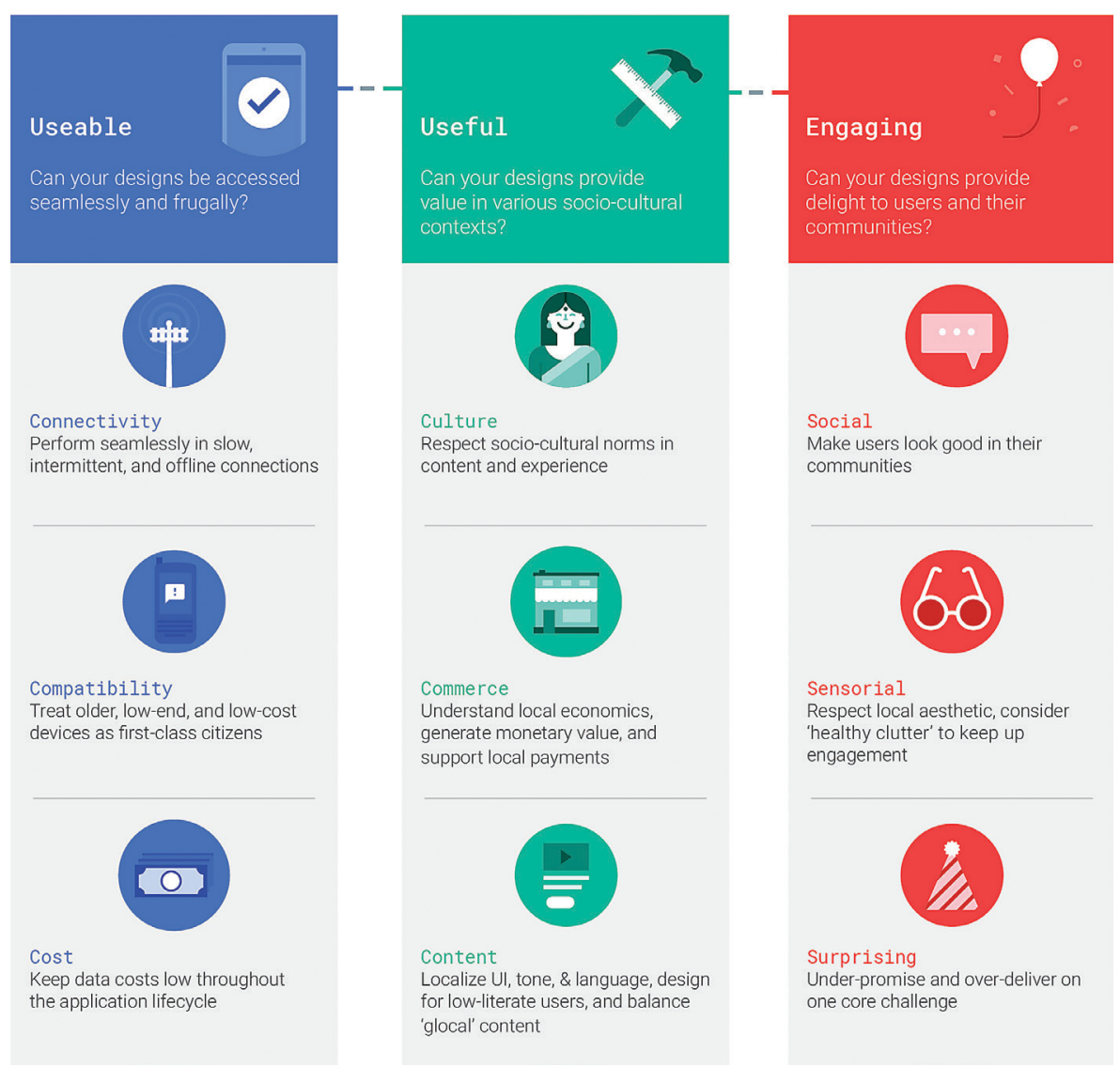

Figure 2. Framework for technology design for emerging markets. 


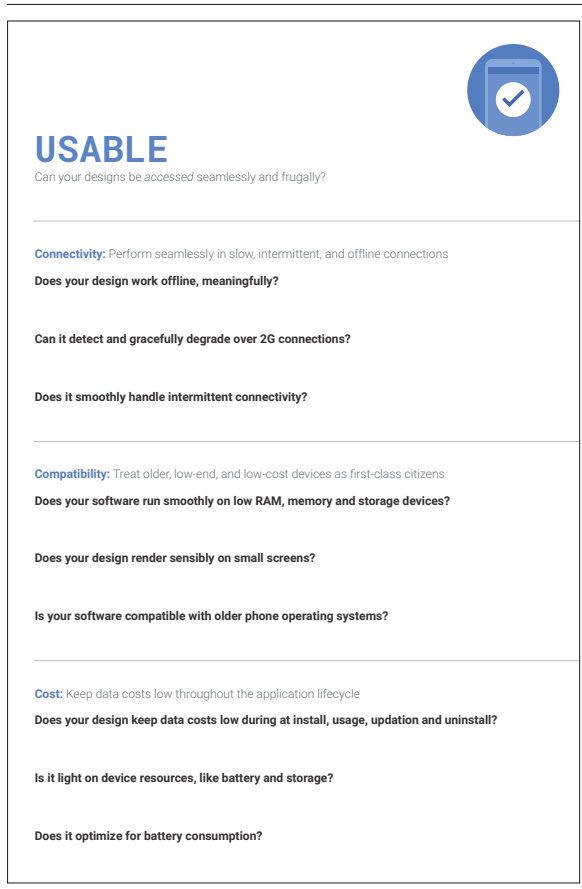

offline. Consider the fact that 95 percent of the Global South has $2 \mathrm{G}$ coverage, but only 69 percent has $3 \mathrm{G}$ coverage (as low as 44 percent in Sub-Saharan Africa), and a small minority has $4 \mathrm{G}$. During our research studies in Thika, Kenya, we met Faith, a mobile-phone-shop employee. Faith goes to phone settings and manually toggles data off to cut down on data costs. She does this about five to seven times a day. Many broadband users we have met unplug or turn off their routers regularly at night, since broadband is capped and expensive. In contrast to the turn toward innovation for always-on infrastructures, such as cloud computing, the Internet of Things (IoT), or contextual services, the network is never a constant in emerging markets. Can your (visual, interaction, and motion) design perform meaningfully and seamlessly in $2 \mathrm{G}$ with intermittent connections? Does your design treat

\section{Can your design be compatible with the hardware and software of low-end devices?}

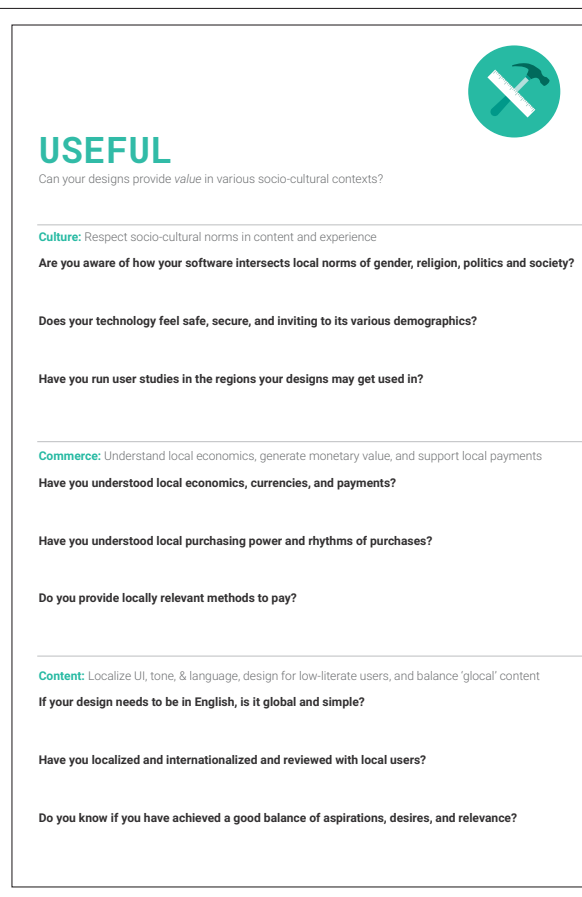

the lack of connectivity (offline) as a starting point?

Compatibility. Smartphones have increased access to the Internet, but nearly one-third of the devices sold today in countries like India and Indonesia have low-end specifications, costing between $\$ 40$ and $\$ 60$ [1]. Characteristics include $512 \mathrm{MB}$ internal memory, small screen sizes, low resolution, and low battery life. Low-cost devices are meant to last at least five years [2]. Many devices often run on older operating systems, due to longer life spans of devices, gifting from urban to rural segments, and second-hand marketplaces in emerging markets. Shekar in the Theni district works as an autorickshaw driver in Chennai, gifting his older phones to his siblings back home when he upgrades to a new model. Can your design be compatible with the hardware and software of low-end devices? Better still, can it make low-end specs and older OS versions first-class citizens of the experience?

Cost. Data costs can be a substantial proportion of monthly expenses, as high as 5 percent of income in Nigeria and 11 percent in Uganda. According to an Internet. org report, in order to reach half the world, an app's monthly mobile data consumption should be no more than 250MB [3]. Types of sacrificing

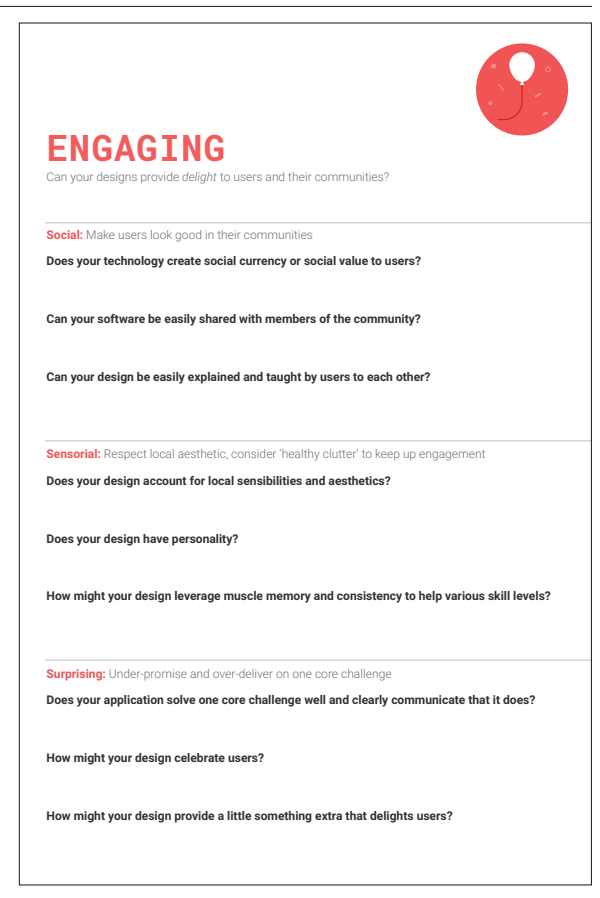

behavior, such as foregoing basic needs to purchase mobile credit, were noted in one in five participants in a survey among lowincome phone users in Kenya [4]. Online software and applications should be sensitive to keeping data consumption low throughout the app lifecycle, from installation to usage to updates to background services. Furthermore, application updates are often ignored by mobile users due to high costs and low relevance, as seen in our research. Can your application gain user trust by being transparent about costs, providing smart defaults, and giving users control? Can you keep battery consumption low, because electricity is not reliable and often expensive or supplemented through charging stations in many emerging markets?

Next, does your technology provide value to those who may consider it? Is there a sociocultural fit?

Culture. Have you conducted user research in the regions of interest to know what the existing social, cultural, political, and religious norms are, and how your design intersects with these vectors? Is your content friendly to various user groups? As an example, during a conversation with Rani, a 36-year-old housewife, about YouTube content in an orthodox Hindu family in Lucknow, a pop-music video started 
auto-playing. She viewed the video as offensive adult content, becoming concerned about her children's safety online. In another example, we were considering a mobile application for on-the-go consumption in Manila's train stations. We quickly learned that stations were considered risky and insecure; most passengers did not take their devices out of their pockets. If men are more likely to own and use technologies [5], how might you consider women in your designs? Human-powered systems are often better and more robust than automated systems in emerging markets - how might you then reimagine access?

Commerce. Local economies may vary from place to place. Does your design account for local practices in buying, selling, or saving, as applicable? Financial inclusion via banking and savings are a challenge in many nations; cash rules around the world. While plastic money is used by only a minority in urban middle classes, mobile money and carrier payments are making inroads in many areas. Cash on delivery (CoD) is increasingly used to enable easier cash payments and verification of deliveries before payments. Social media is used by many small to midsize businesses (SMBs) and traders to market and sell products. If your design involves payment, does it support locally relevant forms of payment? Does it support local business models? Seasonalities such as festivals and culturally significant events may impact the ebb and flow of expenditure.

Content. Have you localized and internationalized for the right languages? Has the local content been user tested? Localized content is often viewed as unnatural, stiff and formal, as failing to sound like locals' everyday speech. Are you using global English that avoids Western centricity? One of the onboarding screens for our products originally welcomed users in India with "new awesomeness ahead"-the American slang term was then replaced with Indian-English text. Find your place on the local-global content spectrum: Hyperlocal content may be relevant, but global content may be aspirational. If you anticipate usage by those with lower literacy, design interactions and navigation accordingly by minimizing text input and increasing "tappability," adding visual richness and minimizing hierarchy.

Finally, even if the design is easy to use and useful, does it fit into desires, practices, and values?

Social. The importance of meaning-making and gaining a sense of belonging through social units may be more pronounced in some emerging markets. Limited resources may mean that individual tasks are shared and that there is some emphasis on delighting each other, not just oneself. Resources may be shared, intermediated, and pooled, including devices and usage. Our research studies show us the importance of word-of-mouth and trust in peers to take up a new technology. Can your design create valuable social currency that makes users look good in their groups and community? How might the social infrastructure play a part in your designs?

Sensorial. Emerging-market contexts are often vibrant, busy, and densely populated. Engagement with the immediate environment through multiple senses (visual, aural, olfactory, and tactile) may be more pronounced. Today's Western aesthetics, such as minimal use of color, sound, and text, and stylized visual elements often fade out in these environments. How might one create the right amount of "healthy clutter" to balance clear, actionable user interfaces and local needs for visual density and vibrancy?

Surprising. The daily experience can be challenging in the Global South, from satisfying basic needs to standstill traffic to economic and infrastructural constraints. To help create escapes from these constraints, deeply appreciate elements of your designs that attempt to delight and surprise. Focus on one core pain point that your product addresses, and see if you can solve it really well. Then, can you provide a little extra something that allows users to celebrate their relief?

\section{ENDNOTES}

1. GfK Point of Sales. 2015; http://www.gfk. $\mathrm{com} /$ solutions/point-of-sales-tracking/ point-of-sales-tracking/

2. Canalys smartphone projections. 2016; https://www.canalys.com/chart/index.html

3. Internet.org. State of connectivity report. 2015; https://fbnewsroomus. files.wordpress.com/2016/02/state-ofconnectivity-2015-2016-02-21-final.pdf

4. Okune, A. Understanding "foregoing” behavior at the base of the pyramid (BoP) in Kenya. 2013; https://ihub.co.ke/blogs/13128

5. GSMA. Bridging the gender gap: Mobile access and usage in low and middle-income countries. 2015; http://www.gsma.com/ mobilefordevelopment/wp-content/ uploads/2016/02/Connected-WomenGender-Gap.pdf

\section{(1) Nithya Sambasivan is a researcher} in Google's Next Billion Users team who previously worked at Google Access and Google.org. She has a Ph.D. in informatics from the University of California, Irvine, and an M.S. in human-computer interaction from Georgia Tech. Her research has won many awards at CHI and ICTD.

$\rightarrow$ nithyasambadagoogle.com

(1) Nibha Jain is the co-founder and research lead of YouTube's Emerging Markets initiatives. She enjoys developing new frameworks, guidelines, and research tools and techniques that can help teams better understand their users. She has an M.S. in industrial design from Georgia Tech and an undergraduate degree from National Institute of Design, India. $\rightarrow$ nibhalagoogle.com

(1) Garen Checkley is the co-founder of YouTube's Emerging Markets initiatives. He led the design of the first version of YouTube Go, a new app designed and built specifically for the next billion users. Currently, he evangelizes product design methods for new Internet users to UX, product, engineering, and marketing teams across Google.

$\rightarrow$ garenagoogle.com

(1) Asif Baki leads research at Next Billion Users at Google. He has a master's in $\mathrm{HCl}$ from the University of Michigan, Ann Arbor, and an undergraduate degree in economics and computer science from the University of Illinois at Urbana Champaign. Prior to Google, he worked at Microsoft on digital media experiences.

$\rightarrow$ asifbakidgoogle.com

(1) Taylor Herr is a visual designer in Google's Next Billion Users team. She especially enjoys projects that are illustration- or brandingfocused. She has an undergraduate degree in visual communications.

$\rightarrow$ therragoogle.com 